\title{
Desmoplastic Variant of Ameloblastoma: A Rare Case Report and Review of Literature
}

\section{Manthru Naik $\mathbf{R}^{1}$, Sridhar Erugula $\mathbf{R}^{2}$, Ayesha $\mathbf{S}^{2 *}$, Jagadeesh $\mathbf{K}^{3}$, Santhosh Kumar PV4, Roger Paul T5, Bhogavaram B ${ }^{6}$, Shahid $I^{7}$ and} Anusha $\mathrm{E}^{8}$

${ }^{1}$ Department of Oral and Maxillofacial Surgery, Govt. Dental College and Hospital, India ${ }^{2}$ Department of Oral Pathology and Microbiology, MNR Dental College and Hospital, India ${ }^{3}$ Department of Conservative Dentistry and Endodontics, Government Medical College and Hospital, India ${ }^{4}$ Department of Oral and Maxillofacial surgery, Government Dental College and Hospital, India ${ }^{5}$ Department of Oral and Maxillofacial Surgery, Lenora Dental College, India ${ }^{6}$ Department of Oral and Maxillofacial Surgery, Sri Sai College of Dental Surgery, India ${ }^{7}$ Department of Oral Medicine and Radiology, Sparkle Dental Hospital, India ${ }^{8}$ Department of Pedodontics, Govt. Dental College, India

*Corresponding author: Ayesha Sameera, Department of Oral Pathology and Microbiology, MNR Dental College and Hospital, Hyderabad, Telangana, India, Email: seashell.ayesha@gmail.com

\section{Abstract}

Desmoplastic ameloblastoma (DA) is a very rare variant of ameloblastoma with distinctive clinical, radiological and histological features. The current article focuses on a DA which occurred anterior to premolar region of the right mandible in a 28 year-old women. Radiographs showed mixed radiopaque - radiolucent lesion with buccal cortical plate expansion. Histological examination showed large extensive stromal desmoplasia with small tumour nests of odontogenic epithelium scattered in stroma. A marginal resection sparing the lower border of the mandible was performed from teeth 31 to 45 . The patient is currently free of disease and undergoing routine follow-up.

Keywords: Desmoplastic ameloblastoma; Female; Mandible; Marginal resection

\section{Abbreviations: DA: Desmoplastic ameloblastoma}

\section{Introduction}

Ameloblastoma is a benign neoplasm derived from enamel organ. Several histologic subtypes of this tumour have been described. The desmoplastic variant was first described by Eversole, et al. [1] in 1984 as "ameloblastoma with pronounced desmoplasia". Till date 170 cases have been reported [2]. This unusual variant is characterized histologically by extensive stromal collagenisation/desmoplasia with small nests and strands 
of odontogenic epithelium suggesting its origin from de novo synthesis of extracellular matrix proteins [3]. In 2005, the World Health Organization classification of odontogenic tumours adopted DA as a histologic type of ameloblastoma with distinct clinical, radiologic, and pathologic features [4]. Waldron \& El Mofty [5] in 1987 described a hybrid type and conventional ameloblastoma, which they suggested represented a "collision tumour."

Radiographically most of the DA shows an image suggesting a benign fibro-osseous lesion [6]. Limited cases of DA have been reported in the literature with very few cases described in Indians. This article aims to describe a case of DA which occurred in the mandible of a 28 year-old female patient along with providing additional clinical, radiographic and histological data.

\section{Case Report}

A 28-year-old female patient presented with the chief complaint of a painless progressive swelling in the right mandibular labial vestibule, in the region of lateral incisor and first premolar teeth. There was no history of trauma to that area. No relevant medical history .The swelling was noticed 6 months ago and was palpable extraorally below the right angle of the mouth. There was no evidence of cervical lymphadenopathy. Intraoral examination revealed a nonmobile, firm, well-defined, $2 \times 2$ $\mathrm{cm}$ swelling that located in buccal vestibule extending from teeth 41 to 44 . The adjacent teeth were vital and nonmobile (Figure 1). Displacement of 42 and 44 was noted. Bilateral mandibular canines were missing without any history of extraction.

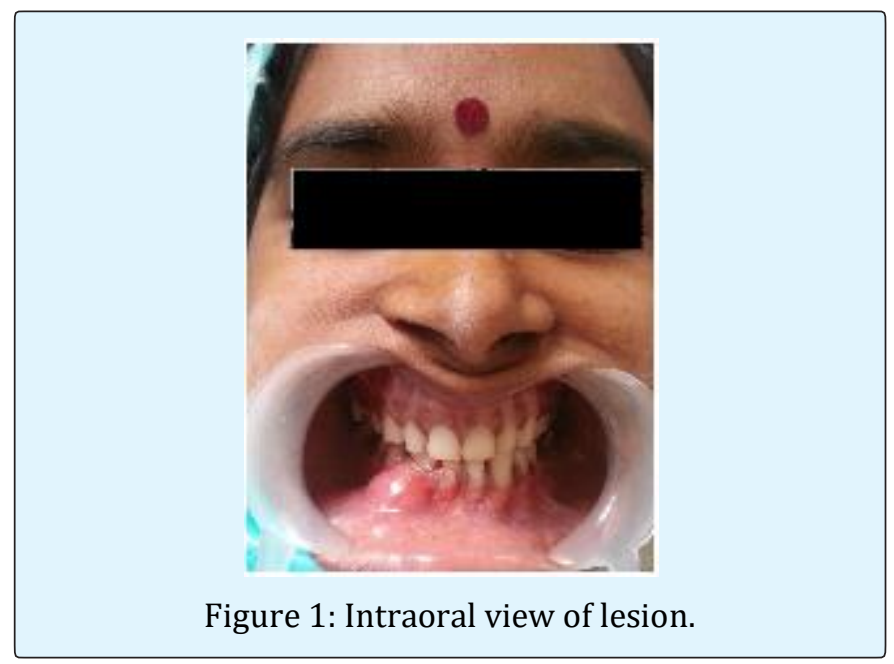

Orthopantomogram revealed a non corticated, pear shaped, mixed radiopaque and radiolucent lesion with indistinct borders involving right parasymphyseal region of mandible (Figure 2a). The lesion extended from the alveolar crest to the region between the apices of the roots of the lateral incisor and first premolar teeth. The roots of these teeth were diverged but root resorption was not evident. The periodontal ligament spaces appeared to be intact. Mandibular occlusal view (Figure $2 \mathrm{~b}$ ) and CT axial view (Figure 2c) revealed expansion and perforation of buccal cortical plate. Needle aspiration failed to yield fluid.
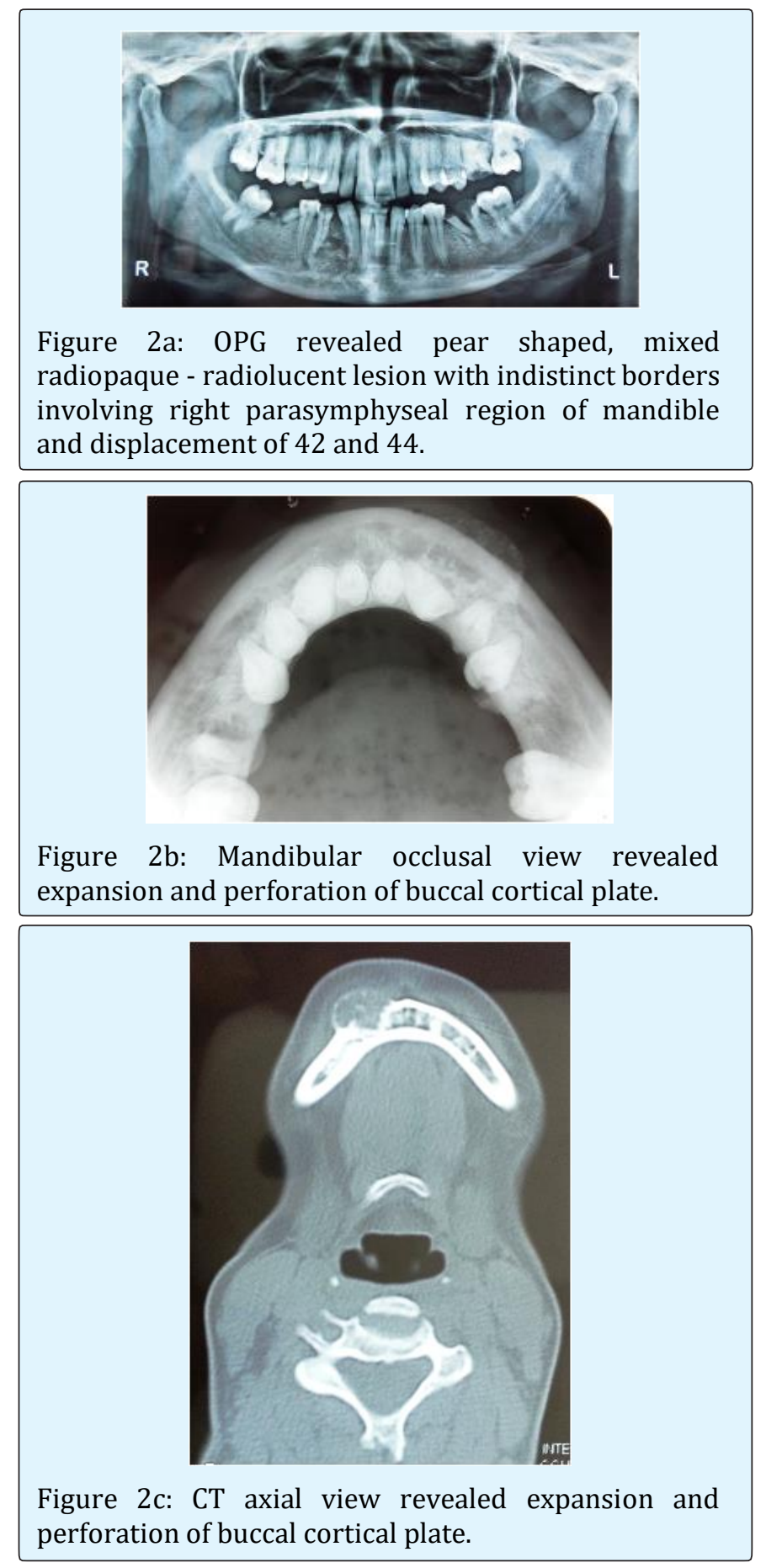
With a provisional diagnosis of benign odontogenic tumour or fibro-osseous lesion, an incisional biopsy was made to obtain a definitive diagnosis. Microscopic examination of the surgical specimen showed narrow, slender, irregular odontogenic islands with stretched-out 'kite-tail' appearance in a moderately cellular fibrous connective tissue with abundant collagen formation (Figure 3a). Extensive desmoplasia was seen throughout the stroma compressing the epithelial islands. The epithelial islands were made up of loosely arranged spindle cells with peripherally palisaded tall columnar to cuboidal hyperchromatic cells (Figure $3 b$ ). Some nests demonstrated central cystic degeneration. Osseous metaplasia was not seen. Immunohistochemistry showed positive staining for pancytokeratin, CK 8, 13 and 19 (Figures 3c \& 3d). A diagnosis of DA was made.

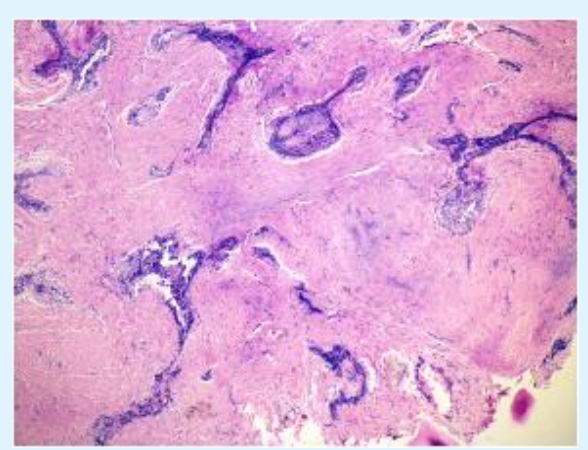

Figure 3a: Narrow, slender, irregular odontogenic islands with stretched-out 'kite-tail' appearance in a densely collagenized stroma. Extensive desmoplasia was seen throughout the stroma compressing the epithelial islands. (H\&E stain, $\times 10$ ).

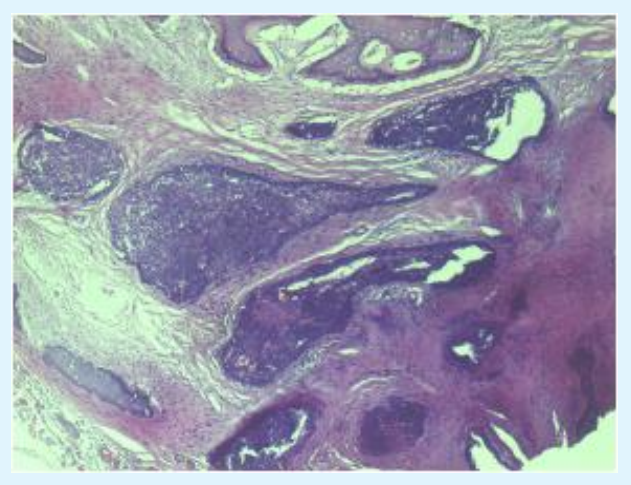

Figure 3b: Epithelial islands were made up of loosely arranged spindle cells with peripherally palisaded tall columnar to cuboidal hyperchromatic cells. Some nests demonstrated central cystic degeneration. Some peripheral non-neoplastic bone was seen without osseous metaplasia. (H\&E stain, $\times 10$ ).
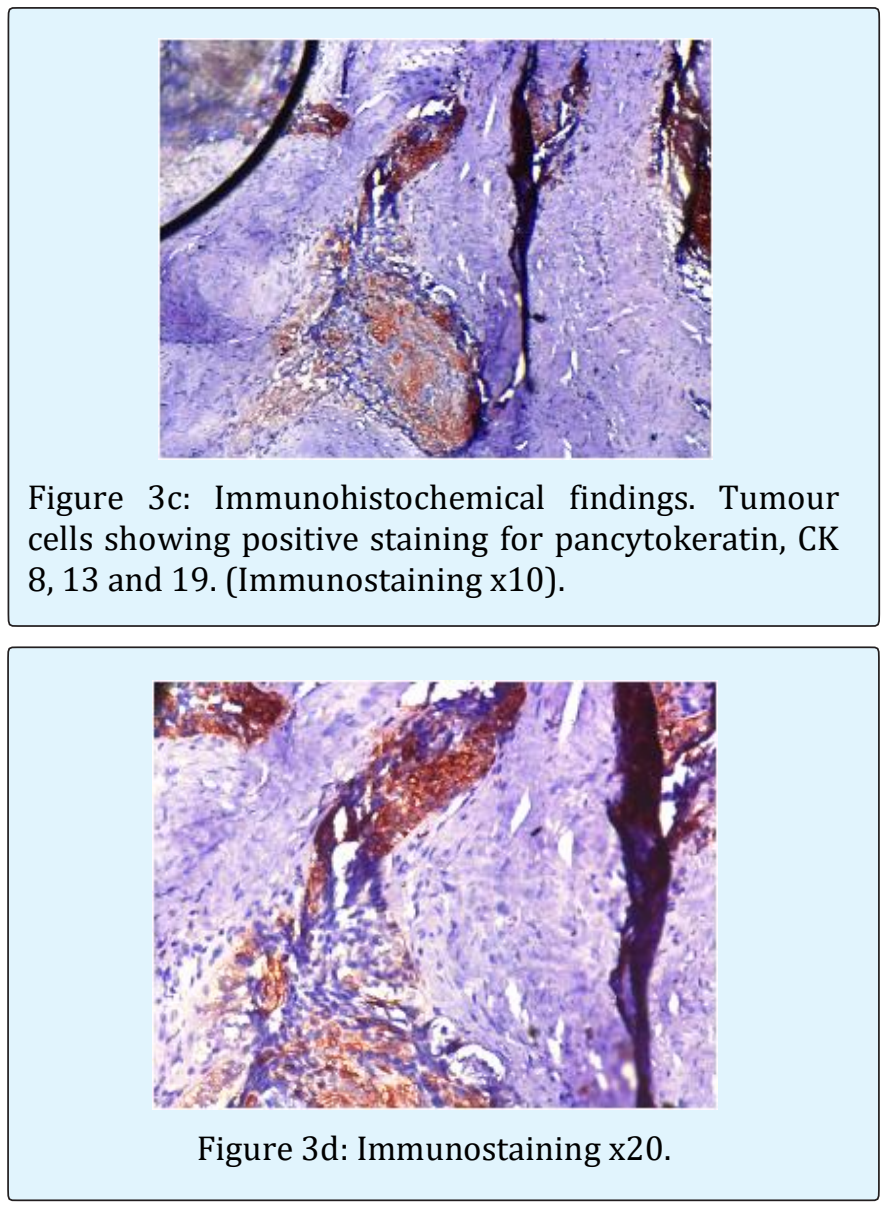

The patient was treated with marginal resection from teeth 31 to 45 , maintaining the lower border of the mandible via lower lip split approach under general anesthesia. A $2.4 \mathrm{~mm}$ reconstruction plate was fixed to minimize the risk of fracture. The postoperative period was uneventful. The missing teeth were rehabilitated by fixed partial dentures. The patient is undergoing routine follow-up without any signs of recurrence after 16 months (Figures 4a-f).

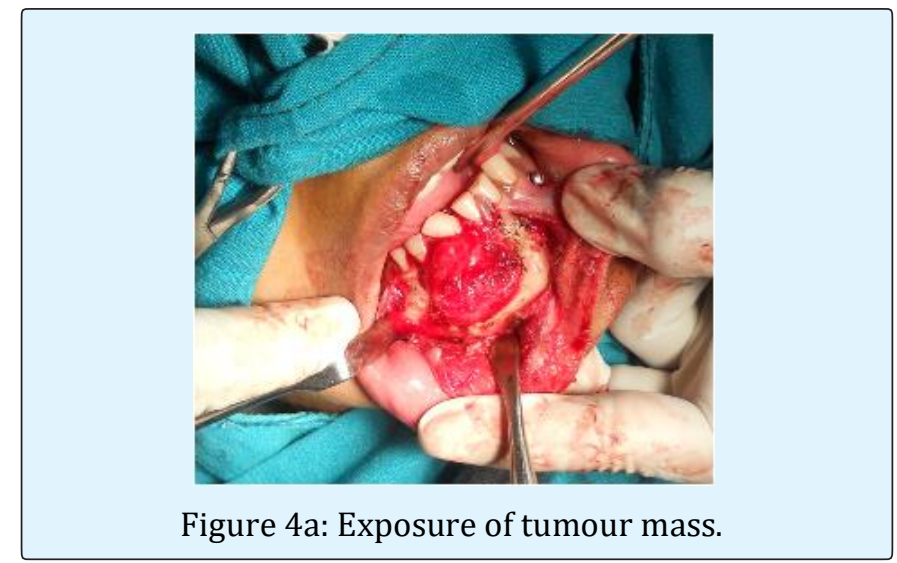



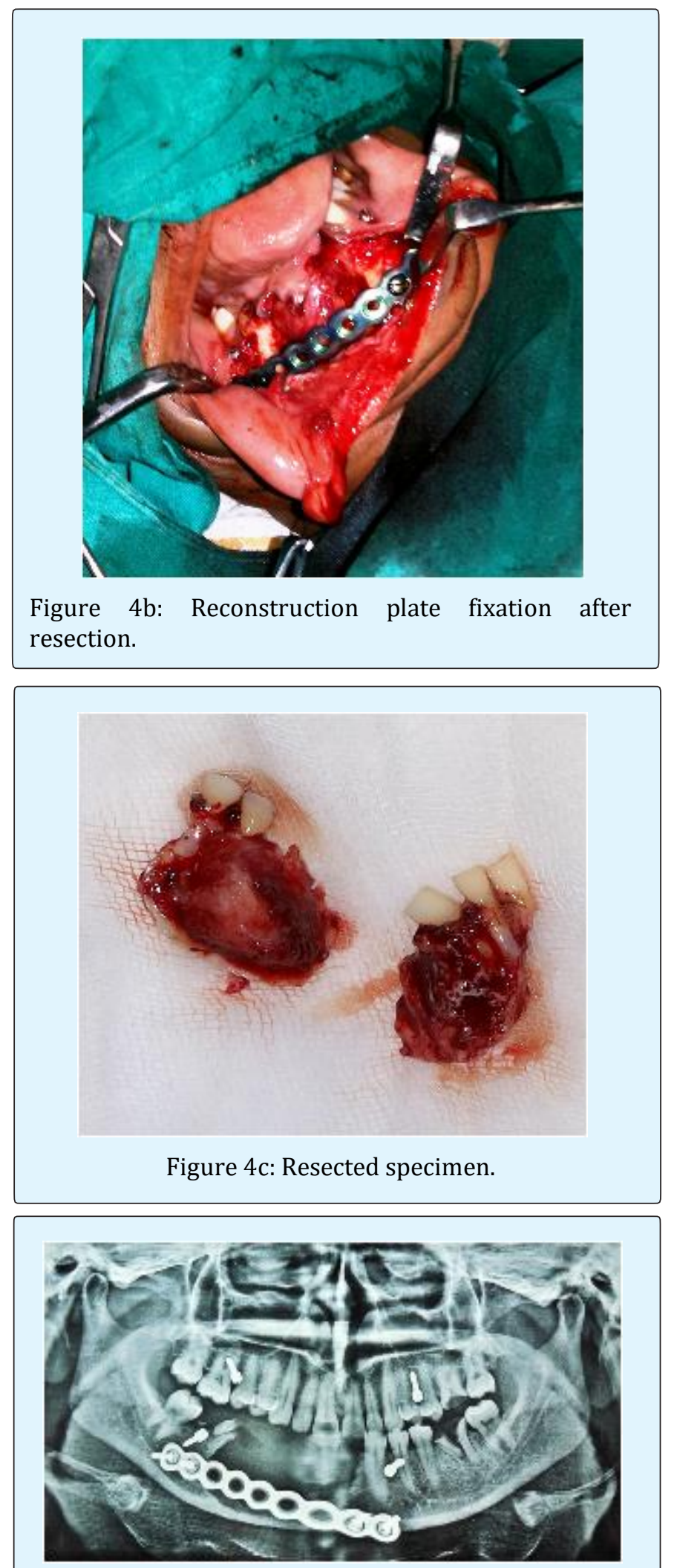

Figure 4d: Post-operative OPG.
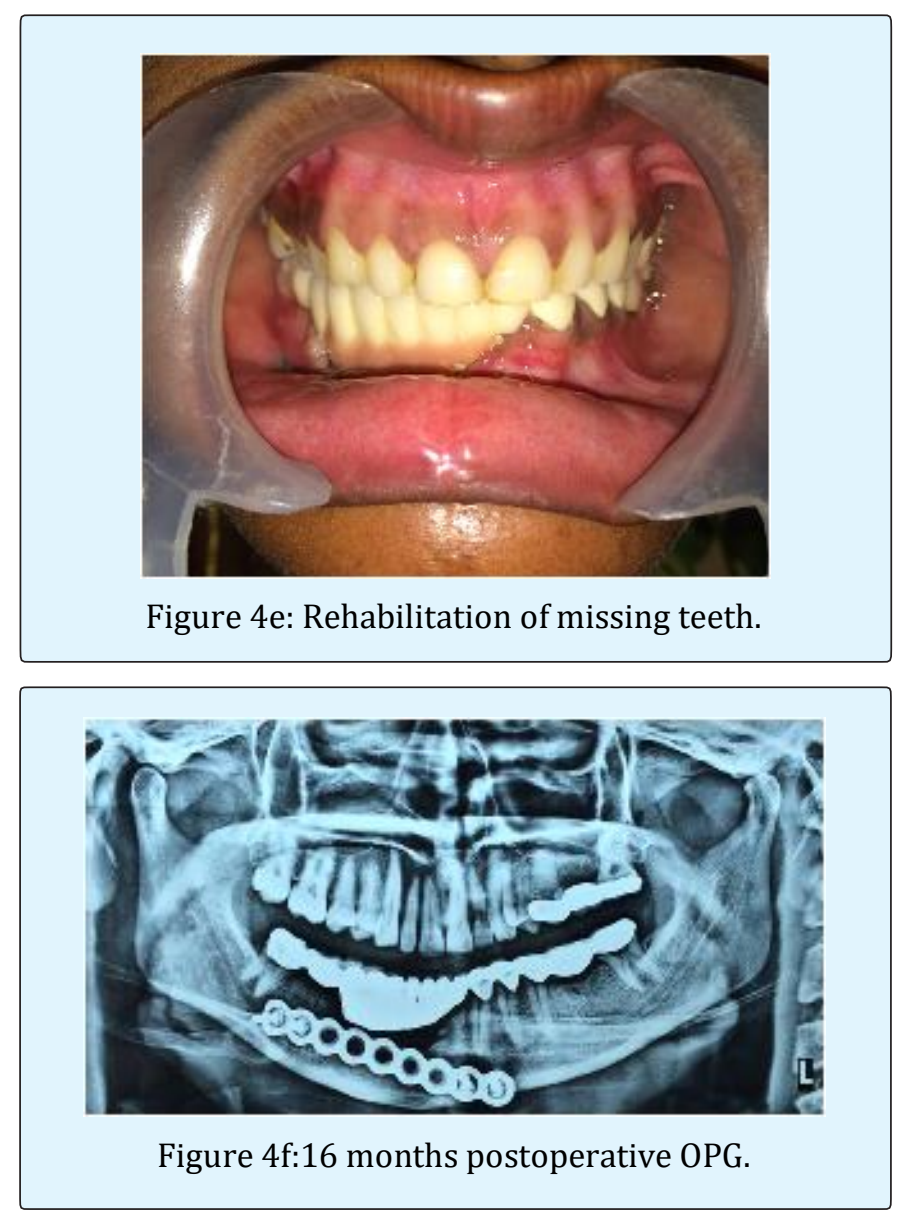

\section{Discussion}

DA is a rare entity of ameloblastoma, with an incidence of $0.9 \%$ to $13 \%$ among all ameloblastoma cases [6,7]. Clinically, DA may develop in all ages, however, people of the 4th and 5th decade are more prone to be affected and no gender predilection is reported [6]. DA involves maxilla and mandible equally unlike other types of ameloblastoma where they show marked predilection for the posterior mandible (5:1, mandible: maxilla).

It is generally agreed that an ameloblastoma has the potential to arise from the epithelial rests of Malassez, reduced enamel epithelium that envelops impacted teeth, or from the gingival epithelium. But on the coronal CT imaging, DA lesions show continuation with the periodontal membrane space of adjacent teeth. Oxytalan fibers are characteristically seen in the periodontium, were demonstrated in the stroma of DA by Kishino, et al. [8]. On the basis of the radiographic and histologic findings, DA is believed to be developed from the epithelial rests of Malassez in the periodontal membrane of the related tooth [9]. 


\section{Open Access Journal of Dental Sciences}

Clinically, DA is a painless swelling, frequently with buccal expansion [6]. Maxillary lesions are generally more dangerous than mandibular lesions as they tend to invade the adjacent sinus, orbit and involving vital structures as the maxillary bone is a thin therefore forms a weak barrier for tumours when compared to the thick mandible [10]. Tooth displacement is a common feature in DA $[11,12]$. Similarly, the present case of DA is located in the anterior- premolar region of the mandible as a painless swelling with buccal cortical expansion and tooth displacement.

Keszler, et al. [13] reported a higher recurrence rate of DA $(21.4 \%)$ than the other type $(10.1 \%)$ of ameloblastoma. This may be due to potential to grow to a large size and bone invasion [10]. Desmoplastic ameloblastoma have a very distinctive radiographic presentation. Uniformly it is found to be a mixed radiolucent- radiopaque mass with indistinct borders on the plain radiograph. In contrast to other ameloblastomas that has a unicystic, multilocular, or soap-bubble appearance [14]. The mixed radiographic appearance of DA is due to osseous metaplasia within the dense fibrous septa that characterizes the lesion [15]. But, Takata, et al. [16] attributed the mixed radiologic appearance and ill defined margins of DA to its infiltrative nature [16].

According to Philipsen, et al. [17] radiographically illdefined borders are suggestive of an infiltrative process with propensity to recur. CT in high resolution clearly defines the tumour boundaries and extension to the adjacent structures along with the ability to distinguish desmoplastic ameloblastoma from other fibro-osseous lesions by presence of thick bony trabeculae situated peripherally between the tumour elements [14].

The panoramic radiograph, CT and MRI images of DA are not very specific as they can mimic the radiographic appearance of chronic sclerosing osteomyelitis, fibrous dysplasia and when well-circumscribed an ossifying fibroma [10]. Histopathologically, ameloblastomas (solid / multicystic) contain two main histopathology types; follicular and plexiform. These consist of proliferating, irregularly shaped islands of the narrow cords of odontogenic epithelium in a connective tissue stroma [10]. By contrast, DA is composed of small islands of tumour cells located within an extensively collagenous stroma (desmoplasia) [7]. The mechanism of desmoplasia is not understood completely. Immunohistochemical studies showed variable expression of $\mathrm{S}-100$ protein and desmin, high expression of caspase- 3 and Fas, decreased expression of cytokeratin 19 and high expression of p63 [15]. Marked expression of transforming growth factor- $\beta$ has been demonstrated and it may play a role in the desmoplasia [16]. It has also been proposed that it may indicate a maturation process and the lesions in the anterior region may mature faster, hence the finding of desmoplasia [15]. The epithelial islands showed central spindle cells with peripherally palisaded columnar to cuboidal cells. This case is consistent with the common features reported in the literature. Typical ameloblastic columnar cells may be scant. Osteoplasia may also be present.

The tumour cells of DA are positive for cytokeratin (CK) - 8, 13, 19; filaggrin and ameloblastoma antibodies [18]. The surgical management of DA has been controversial due to its high rate of recurrence [19]. Sun, et al. [6] reported that enucleation provided a recurrence rate of $21.1 \%$ and resection gave $3.1 \%$. This recurrence originates from small fragments of the tumour left in situ. Therefore resection is the most preferred treatment modality for desmoplastic ameloblastoma.

\section{Conclusion}

The DA is considered as a rare lesion, but information suggests that it can be considered as a distinct lesion. Diagnostician should be alert as DA can have a differential diagnosis ranging from periapical lesion to any fibroosseous lesions and neoplastic growth. The definitive diagnosis requires histopathological examination. DA should always be treated by complete resection and long term follows up as it has potential for recurrence.

\section{References}

1. Eversole LR, Leider AS, Hansen LS (1984) Ameloblastomas with pronounced desmoplasia. J Oral Maxillofac Surg 42(11): 735-740.

2. Sharma Lamichhane $N$, Liu Q, Sun $H$, Zhang $W$ (2016) A case report on desmoplastic ameloblastoma of anterior mandible. BMC Research Notes 9: 171.

3. Ashman GS, Corio RE, Eisele Dw, Michael T Murphy (1993) Desmoplastic ameloblastoma: A case report and literature review. Oral Surg Oral Med Oral Pathol 75(4): 479-482.

4. Barnes L, Eveson EJ, Reichart P, Sidransky D (2005) World Health Organization Classification of Tumours: Pathology and Genetics of Head and Neck Tumours. $3^{\text {rd }}$ (Edn.), Lyon, France: IARC Press; pp: 296-300. 


\section{Open Access Journal of Dental Sciences}

5. Waldron CA, El Mofty SK (1987) A histopathologic study of 116 ameloblastomas with special reference to the desmoplastic variant. Oral Surg Oral Med Oral Pathol 63(4): 441-451.

6. Sun ZJ, Wu YR, Cheng N, Zwahlen RA, Zhao YF (2009) Desmoplastic ameloblastoma-A review. Oral Oncol 45(9): 752-759.

7. Philipsen HP, Reichart PA, Takata T (2001) Desmoplastic ameloblastoma (including "hybrid" lesion of ameloblastoma): biological profile based on 100 cases from the literature and own files. Oral Oncol 37(5): 455-460.

8. Kishino M, Murakami S, Fukuda Y, Ishida T (2001) Pathology of the desmoplastic ameloblastoma. J Oral Pathol Med 30(1): 35-40.

9. Kawai T, Kishino M, Hiranuma H, Sasai T, Ishida T (1999) A unique case of desmoplastic ameloblastoma of the mandible: report of a case and brief review of the English language literature. Oral Surg Oral Med Oral Pathol Oral Radiol Endod 87(2): 258-263.

10. Gopal Sharma, Reema Rao, Preeti Talreja, Mahesh B Pokle, Resha sawla, et al. (2014) Case report of patient with desmoplastic ameloblastoma. Int J Cur Res Rev 6(10): 37-44.

11. Márcio Bruno Amaral, Belini Freire-Maia, Marcella Rezende Serpa, Ricardo Alves Mesquita (2010) A case report of desmoplastic ameloblastoma. J Clin Exp Dent 2(3): 149-152.

12. Shashikanth MC, Neetha MC, Ali IM, Shambulingappa P (2007) Desmoplastic ameloblastoma in the maxilla: a case report and review of literature. Indian J Dent Res 18(4): 214217.

13. Keszler A, Paparella ML, Dominguez FV (1996) Desmoplastic and non-desmoplastic ameloblastoma: a comparative clinicopathological analysis. Oral Dis 2(3): 228-231.

14. Patrick J Louis, Richard C Fugler, Meredith August (2000) Mixed Radiolucent/Radiopaque Lesion of the Maxilla. J Oral Maxillofac Surg 58(1): 86-90.

15. Yazdi I, Seyed Majidi M, Foroughi R (2009) Desmoplastic ameloblastoma (a hybrid variant): report of a case and review of the literature. Arch Iran Med 12(3): 304-308.

16. Takata T, Miyauchi M, Ogawa I, Kudo Y, Takekoshi T, et al. (2000) Immunoexpression of transforming growth factor beta in desmoplastic ameloblastoma. Virchows Arch 436(4): 319-323.

17. Philipsen HP, Ormiston IW, Reichart PA (1992) The desmo and osteoplastic ameloblastoma. Histologic variant or clinicopathologic entity? Case reports. Int J Oral Maxillofac Surg 21(6): 352-357.

18. Kumamoto H, Kamakura S, Ooya K (1998) Desmoplastic ameloblastoma in the mandible: Report of a case with an immunohistochemical study of epithelial cell markers. J Oral Med Pathol 3(1): 45-48.

19. Nakamura N, Higuchi Y, Mitsuyasu T, Sandra F, Ohishi M (2002) Comparison of long-term results between different approaches to ameloblastoma. Oral Surg Oral Med Oral Pathol Oral Radiol Endod 93(1): 13-20. 